Angesichts der Fülle widerstreitender Kräfte konnte der Führerwille, auch wenn er anderes im Sinne gehabt hätte, schließlich gar nicht mehr anders als jeweils nur unzusammenhängend und abrupt von Fall zu Fall Anstöße in diese oder jene Richtung geben, war aber außerstande, die sich daraus jeweils entwickelnden neuen Organisationen, Kompetenzen und Ambitionen zu übersehen und im Zaume zu halten. Die institutionellen und rechtlichen Folgen der jeweiligen Führerbefehle und -erlasse wurden immer unabsehbarer, gerieten mit späteren Führerbevollmächtigungen in Widerspruch und lagen selbst noch als politisch entleerte Organisationshülsen der Einheitlichkeit und Regelhaftigkeit der Machtausübung und Herrschaftsorganisation störend im Wege. ${ }^{32}$

Angesichts der analytischen Erkenntnis, dass Hitler keineswegs der »Herr und Meister« des Nationalsozialismus gewesen sei und mithin die Geschichte des >Dritten Reiches nicht ausschließlich auf die Person Hitler zu reduzieren sei, bewertete Broszat auch dessen aktive Rolle und direkte Hauptverantwortung bei der Vernichtung der europäischen Juden geringer und kam zu einer »neuen« Einschätzung. Seiner Auffassung nach konnte Hitler nämlich bereits zu diesem Zeitpunkt keineswegs mehr »über das $\mathrm{Ob}$, Wann und Wie« bestimmter Maßnahmen »souverän entscheiden «. ${ }^{33}$ Die Massenverbrechen an den Juden waren auch und vor allem das systemimmante Resultat eines sich immer mehr radikalisierenden »Bewegungs «-Regimes. Sie waren aus den strukturellen Bedingungen und aus den »negativen Zielrichtungen der Weltanschauungspolitik« - in deren Fokus der Antisemitismus, der Antibolschewismus und der Gewinn von Lebensraum im »Osten« stand - des NS-Systems zu verstehen. Wie die schrittweise gesetzliche Diskriminierung der Juden in den 1930er-Jahren, so war auch die »Endlösung« für Broszat nicht von vornherein geplant, erfolgte nicht einheitlich und nicht systematisch. ${ }^{34}$

\title{
2. Die Infragestellung der Totalitarismustheorie in der Wissenschaft im Gegensatz zur Totalitarismuskonzeption im Monat in den 1960er-Jahren
}

Auch durch die Ergebnisse der empirischen NS-Forschung verstärkten sich Anfang der 1960er-Jahre in der Totalitarismusforschung die Vorbehalte gegenüber der Totalitarismustheorie. ${ }^{35}$ Wie gezeigt traten bereits in der empirisch ausgerichteten politik- und sozialwissenschaftlichen Forschung unmittelbar nach Stalins Tod infolge des einsetzenden Wandlungsprozesses des sowjetischen Herrschaftssystems Zweifel an der klassischen Totalitarismustheorie auf. Zentrale Definitionskriterien für die Analyse des Stalinismus passten nicht mehr für den Nachstalinismus, da nicht zuletzt der Terror als totalitarismustypisches Phänomen in der Ära Chruschtschows seine konstitutive Bedeutung verloren hatte. Im Mittelpunkt der Kritik stand der an einem statischen Modell ausgerichtete idealtypische Begriff der totalitären Herrschaft von Carl

32 Ebd., S. 439.

33 Ebd., S. 440.

34 Ebd., S. 437.

35 Vgl. Gerhard Schulz, Der Begriff des Totalitarismus und der Nationalsozialismus, in: Soziale Welt 12 (1961), H. 2, S. 112-128. 
J. Friedrich, an dessen Ende durch methodologisch abgesicherte Untersuchungen die grundsätzliche Verabschiedung der Totalitarismustheorie durch Peter Christian Lutz stand. ${ }^{36}$

Diese sowohl theoretische als auch politische Aspekte umfassende Diskussion über die wissenschaftliche Anwendbarkeit des Totalitarismusbegriffs für die Analyse des Nachstalinismus einerseits und zur gemeinsamen Identifizierung des sowjetischen Kommunismus und des deutschen Nationalsozialismus andererseits spielte im Monat explizit und implizit eine Rolle. Nachdem bereits nach Stalins Tod und nicht zuletzt nach dem XX. Parteitag der KPdSU sowie Chruschtschows Geheimrede im Februar 1956 bei der Analyse des Nachstalinismus unter den Autoren eine Auseinandersetzung über die Kernfrage geführt wurde, inwiefern das Herrschaftssystem noch als totalitär charakterisiert werden könne, kam Martin Jänicke 1966 vor dem Hintergrund des Wandlungsprozesses des sowjetischen Systems auf ein für ihn grundsätzliches Problem der Totalitarismustheorie von Friedrich zu sprechen. Anlässlich der Rezension der von Friedrich allein überarbeiteten und revidierten Neufassung der amerikanischen Ausgabe seiner Totalitarismusstudie war Jänicke der Auffassung - unter anderem aufgrund der Tatsache, dass es Friedrich nur durch eine "willkürliche« Ausweitung des Terrorbegriffs möglich war, dieses entscheidende Totalitarismuskriterium beizubehalten -, dass es dem Autor »nicht nur um die Beschreibung des Totalitarismusphänomens, sondern auch um den Nachweis seiner aktuellen Bedrohlichkeit ging « ${ }^{37}$.

Damit war im Selbstverständnis der westlichen liberal-demokratischen Staaten in den Jahren des Kalten Krieges insbesondere der sowjetische Kommunismus in seiner nachstalinistischen Phase gemeint und implizierte eine politisch-normative Funktion. Denn nachdem der deutsche Nationalsozialismus sowie der italienische Faschismus besiegt waren, erschien der Nachstalinismus als Prototyp der antidemokratischen Herrschaftsform. ${ }^{38}$ Insofern wurde die Totalitarismustheorie für politische Zwecke operationalisiert und diente aktuell einer antikommunistischen Funktion. ${ }^{39}$

36 Siehe hierzu die entsprechenden Ausführungen im Einleitungskapitel.

37 Martin Jänicke, Revision einer Theorie, in: Der Monat 18 (1966), H. 208, S. 78-81, hier S. 80 (Rezension). Die ursprünglich 1956 veröffentlichte Untersuchung erschien, wie bereits gesagt, aufgrund von theoretischen Differenzen zwischen den beiden Autoren in seiner deutschen Ausgabe 1957 in der alleinigen Verantwortung von Friedrich.

38 Das im Laufe der 1950er-Jahre in der Totalitarismusforschung auch besonders der chinesische Kommunismus unter Mao sowie nach der kubanischen Revolution 1959 auch das »Castro-Regime« in den Mittelpunkt rückte, sei an dieser Stelle nur erwähnt, spielt allerdings in der vorliegenden Untersuchung keine besondere Rolle.

39 Indes ist mit Blick auf die klassische Totalitarismustheorie von Carl J. Friedrich, die im Laufe der Jahre von dem deutsch-amerikanischen Politologen immer wieder in einer neuen Version präsentiert wurde, noch hinzuzufügen, dass es sich nach Auffassung von Hans ]. Lietzmann um keine Demokratietheorie handelte. Der Politikwissenschaftler rekonstruiert die ideengeschichtliche und politische Genese von Friedrichs Totalitarismustheorie und legt dar, dass sein Modell nicht der Cegenüberstellung von Demokratie und totalitärer Herrschaft, sondern vielmehr dem Gegensatzpaar von totalitärer und konstitutioneller Diktatur entspricht - und zwar wie es in der besonders von Carl Schmitt geprägten Verfassungsdiskussion der Weimarer Republik gang und gebe war. Wie viele andere deutsche Staatsrechtler ging es Friedrich am Ende der Weimarer Republik darum, Argumente zur Rechtfertigung einer Ausweitung der exekutiven Machtbefugnisse zu finden, von der er sich die Kontinuitätssicherung der politischen Verhältnisse erhoffte. Um die Erosion der staatlichen Herrschaft durch demokratischen Pluralismus und Parlamentarismus aufzuhalten, plädierte Friedrich für eine kons- 
In diesem Zusammenhang existierten Parallelen zum Monat in den 1960er-Jahren. Zwar wurden hier, wie gesagt, durch Jänicke die gravierenden theoretischen Schwächen der friedrichschen Totalitarismustheorie beim Vergleich des Nationalsozialismus mit dem Sowjetkommunismus eingeräumt, indes am Konzept des Totalitarismus festgehalten: sowohl in der vergleichenden Perspektive auf die neuartigen totalitären Diktaturen in Deutschland und der Sowjetunion als auch bei der genuinen Analyse der poststalinistischen Ausprägung kommunistischer Herrschaft. Für diesen Ansatz standen in Anlehnung an die Totalitarismuskonzeption von Brzezinski und an seine früheren Arbeiten insbesondere die von Löwenthal veröffentlichten Beiträge. In seinem Ende 1960 erschienenen einflussreichen Aufsatz Totalitäre und demokratische Revolution ${ }^{40}$ nahm Löwenthal in Anbetracht der nach Stalins Tod einsetzenden Entwicklungen innerhalb des sowjetkommunistischen Herrschaftssystems eine Begriffsbestimmung totalitärer Herrschaft vor, die den neuartigen Regimetypus an Hand der "Machtstruktur und Dynamik« durch die unmittelbare Verbindung eines Konzepts des monopolistischen Parteiregimes mit einem Konzept der Revolution zu begründen versuchte. ${ }^{41}$

Im bewussten Gegensatz zu Arendts Totalitarismusstudie und zu der von Friedrich und Brzezinski gemeinsam konzipierten statischen Strukturanalyse ${ }^{42}$ bemühte sich Löwenthal um eine dynamische Variante des Totalitarismusansatzes und erreichte die Subsumtion des Nationalsozialismus und des Sowjetkommunismus unter ein und denselben Totalitarismusbegriff dadurch, indem er ihn einem spezifischen Revolutionsbegriff zuordnete: nämlich der »Revolution neuen Typs«, das heißt der totalitären Revolution. Diese unterschied Löwenthal ausdrücklich von den bis dahin

titutionelle Diktatur, die sich durch eine vorübergehende Aufhebung der Cewaltenteilung und Suspendierung einzelner Verfassungsbestandteile kennzeichnet, um die Cesamtverfassung zu erhalten oder wiederherzustellen. Zur Rettung der Gesamtverfassung sollen demnach Teile von ihr außer Kraft gesetzt, der Legitimität Vorrang gegenüber der Legalität eingeräumt und eine zeitweilige Diktatur etabliert werden. Dieser etatistische Grundzug zeigte sich nach dem Ende des Zweiten Weltkrieges in Friedrichs Rechtfertigungsversuch der amerikanischen Besatzungsmacht in Deutschland, der zum unmittelbaren Entstehungskontext seiner Totalitarismustheorie gehörte. Denn das Konzept der »totalitären Diktatur«, so Lietzmann, verdankt seine Entstehung nicht, um besonders dessen »Gefährlichkeit oder Abnormalität« zu kennzeichnen, sondern »einem Rechtfertigungsversuch der amerikanischen Besatzungspolitik als einer verfassungsmäßigen (konstitutionellen) Diktatur«. Der Begriff diente dazu, die sowjetische Besatzungsmacht anzuprangern und die US-amerikanische wiederum zu legitimieren (Hans ]. Lietzmann, Von der konstitutionellen zur totalitären Diktatur, in: Söllner/Walkenhaus/Wieland [Hg.], Totalitarismus, S. 174-192, hier S. 177). Für den aktuellen theoretischen politikwissenschaftlichen Diskurs ist für Lietzmann eine wichtige Schlussfolgerung, dass es zukünftig nicht mehr möglich sein sollte, »unter Berufung auf die klassische Totalitarismustheorie naiv eine Alternative von `Totalitarismus $<$ und `Demokratie< zu behaupten. Das Gegensatzpaar, das der klassischen Totalitarismustheorie entspricht, lautet vielmehr: stotalitäre oder konstitutionelle Diktaturı. Von Demokratie ist bei alldem nur am Rande die Rede ... man kann nicht Antitotalitarismus und Demokratie umstandlos für dasselbe halten, wie es sich im Kalten Krieg so schön einbürgerte und nach der deutschen Einheit bisweilen fortgesetzt wird. Die Disjunktion von Totalitarismus und Demokratie ist - gemessen an der Totalitarismustheorie - ein propagandistisch geprägter Wunschtraum und ohne theoretische Grundlage.« (Ebd., S. 191)

40 Richard Löwenthal, Totalitäre und demokratische Revolution, in: Der Monat 13 (1960), H. 146, S. 29-40.

41 Schlangen, Die Totalitarismus-Theorie, S. 71.

$42 \mathrm{Vgl}$. Friedrich/Brzezinski, Totalitarian Dictatorship and Autocracy. 
aus der Geschichte bekannten Revolutionen, speziell von den bürgerlich-demokratischen. Während die demokratischen Revolutionen eine begrenzte Zielsetzung hatten und nach der Etablierung des neuen politischen Systems zum Stillstand kamen, waren die totalitären Revolutionen für Löwenthal dadurch gekennzeichnet, dass sie nach der Machtergreifung permanent versuchten, »die Gesellschaft weiter im Sinne ihrer Ideologie $\mathrm{zu}$ transformieren ${ }^{43}$. Da für Löwenthal die Ideologie geradezu die revolutionäre Umwälzung erforderte und die Partei ihre Macht im Namen der Ideologie rechtfertigte, bedingten sich die »neue Institution der Einparteiherrschaft und die neue Dynamik der sozialen Umwälzung, die gemäß einer vorgegebenen Ideologie von oben gelenkt « wurde, einander gegenseitig. ${ }^{44}$ Charakteristisch sei für totalitäre Regime, dass sie »die Macht nicht nur mit revolutionären Mitteln« eroberten, sondern sie durch »zielbewußtes Inganghalten eines Prozesses gelenkter gesellschaftlicher Umwälzung zu behaupten« suchten - dass sie eine >permanente Revolution von oben anstrebten..$^{45}$

Die Definition des Totalitarismus Löwenthals war also durch die unmittelbare Verknüpfung des strukturellen Aspekts der Einparteienherrschaft sowie durch den dynamischen Aspekt der »revolutionären Ideologie« bestimmt, "weil in diesem Regime der revolutionäre Prozeß selbst zur Institution geworden « war. ${ }^{46}$ Durch diese Klassifizierung gelang es Löwenthal, die totalitären Herrschaftsregime des deutschen Nationalsozialismus und des sowjetischen (sowie chinesischen) Kommunismus von den diversen »revolutionären Diktaturen« des 20. Jahrhunderts zu unterscheiden, die sich für ihn nämlich gerade nicht durch den Versuch auszeichneten, die »Selbstbewegung des revolutionären Prozesses zu verewigen « (Walter Schlangen). ${ }^{47}$

Indes entwickelte Löwenthal seinen Begriff der totalitären Revolution anhand des kommunistischen Revolutionsbegriffs und belegte ihn ausschließlich am Verlauf der Russischen Revolution. ${ }^{48}$ Eine Überprüfung seines dynamischen Totalitarismus-

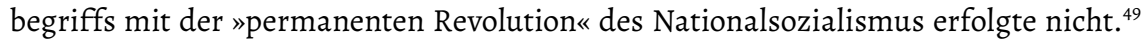
Für Löwenthal stand fest, dass die Russische Revolution, die für ihn ursprünglich starke Parallelen mit den demokratischen Revolutionen aufwies, spätestens seit der Einführung der Neuen Ökonomischen Politik im März 1921 die Tendenz zeigte, die eigene Bewegung zu verewigen, und insofern handelte es sich um »die erste der totalitären Revolutionen unserer Zeit « ${ }^{50}$. Während sich die totalitären Diktaturen für Arendt, Friedrich oder auch Brzezinski durch Terror und politische Säuberungen auszeichneten, umging Löwenthals Konzeption die »Extreme des Grauens«, weil sie nämlich das mit der »Machtübernahme durch eine revolutionäre Partei errichtete diktatorische Herrschaftssystem insgesamt als >totalitär« « begriff. ${ }^{51}$ Daraus ergab sich sowohl eine

43 Richard Löwenthal, Totalitäre und demokratische Revolution, in: Der Monat 13 (1960), H. 146, S. 31.

44 Ebd., S. 32.

45 Ebd., S. 31.

46 Ebd., S. 32.

47 Vgl. ebd., S. 31.

48 Vgl. ebd., S. 33-38.

49 Vgl. bes. Neumann, Permanent Revolution. Zu dem primär anhand des NS-Regimes konzipierten Totalitarismusmodell siehe die entsprechenden Ausführungen im Einleitungskapitel.

50 Richard Löwenthal, Totalitäre und demokratische Revolution, in: Der Monat 13 (1960), H. 146, S. 35.

51 Schlangen, Die Totalitarismus-Theorie, S. 72. 
Ausweitung des Geltungsbereiches als auch eine historisch präzisierende Eingrenzung der Totalitarismuskonzeption von Löwenthal.

Einerseits wurde bereits das institutionelle Grundgefüge der »monopolistischen Parteiregime« als stotalitär <ezeichnet, wodurch es ihm möglich war, den Anwendungsbereich des Begriffes totalitärer Herrschaft ausdrücklich auch auf den sowjetischen Kommunismus in seiner nachstalinistischen Phase anzuwenden; hiermit tat sich ein klarer Gegensatz zu Arendt auf, die 1966 in einem Vorwort zum dritten Band ihrer Totalitarismusstudie mit Blick auf die sowjetische Entwicklung nach Stalins Tod feststellte, dass es »einen echten, wenn auch nie unzweideutigen, Abbau totaler Herrschaft" gab und insofern die empirische Anwendung des von ihr entwickelten Totalitarismusansatzes auf den Nachstalinismus prinzipiell ablehnte. ${ }^{52}$

Andererseits wurden die konkreten Bedingungen mitformuliert, unter denen ein monopolistisches Parteiregime nicht länger als totalitär angesehen werden konnte, wenn sich nämlich der »revolutionäre Charakter der herrschenden Partei im Einparteienregime verändert und das monopolistische Parteiregime seine revolutionäre Dynamik« eingebüßt hatte..$^{53}$ Obwohl es sich bei der »totalitären Parteiherrschaft« Löwenthal zufolge um kein konservatives Phänomen handelte, stand für ihn gleichwohl fest, dass ihr Drang, die Revolution auf Dauer zu stellen, kein vorhersehbares Ende hatte: »Die Totalitären« besaßen »kein Perpetuum mobile, das ihnen den Dauererfolg in der totalen Manipulation der Weltgeschichte sichert[e]«. ${ }^{54}$ Vielmehr wurden unvorhergesehene gesellschaftliche Entwicklungen als Nebenprodukt der "permanenten Revolution von oben « freigesetzt, die ihrerseits der fortgesetzten Durchführung der totalitären Dynamik zuwiderliefen, sodass sich für ihn die grundsätzliche Frage nach den Grenzen der Möglichkeit totaler gesellschaftlicher Planung und nach der Vergänglichkeit des totalitären politischen Systems stellte..$^{55}$

Diese entscheidende Frage seiner Totalitarismuskonzeption aus dem Zusammenhang der Analyse des Sowjetkommunismus war freilich nur empirisch zu beantworten. Dasselbe galt ebenso für die sich aufdrängende Frage, inwiefern das Regime auf der zeitgenössischen Entwicklungsstufe der Veralltäglichung der totalitären Revolution »noch auf den Massenterror zurückgreifen könnte, um einer widerstrebenden Bevölkerung solche Umwälzungen aufzuzwingen«, sodass erst im konkreten historischen Einzelfall analytisch zu prüfen war, ob von einer postrevolutionären »totalitären Parteiherrschaft« gesprochen werden konnte. ${ }^{56}$ Mit anderen Worten: Es war die Frage nach dem »Verhältnis von gesellschaftlichem Entwicklungsstand und totalitärer Bewegung « der KPdSU verbundene Denkansatz, ${ }^{57}$ der es Löwenthal ermöglichte, seine Totalitarismuskonzeption fortan historisch nicht nur im Monat differenziert in erster Linie auf das nachstalinistische Herrschaftssystem anzuwenden. ${ }^{58}$

52 Arendt, Elemente und Ursprünge totaler Herrschaft, S. 475.

53 Schlangen, Die Totalitarismus-Theorie, S. 73.

54 Richard Löwenthal, Totalitäre und demokratische Revolution, in: Der Monat 13 (1960), H. 146, S. 40.

55 Vgl. ebd., S. $39 f$.

56 Ebd., S. 40.

57 Möll, Gesellschaft und totalitäre Ordnung, S. 160; vgl. auch Schlangen, Die Totalitarismus-Theorie, S. 73.

58 Vgl. bes. Richard Löwenthal, Nachtrag 1966 zu ders.: Totalitäre und demokratische Revolution, in: Seidel/Jenkner, Wege der Totalitarismus-Forschung, S. 379-381; ders., The Model of the Totalitarian 
In seinem im Novemberheft des Jahres 1970 erschienenen Beitrag Entwicklung kontra Utopie. Das kommunistische Dilemma ${ }^{59}$ setzte sich Löwenthal rückblickend mit der sowjetischen Entwicklung in den 1960er-Jahren auseinander. Seiner Diagnose nach hatte sich in diesem Zeitraum die »Logik der Ökonomie« gegenüber der »Logik der Ideologie« durchgesetzt, was gleichbedeutend war mit dem Nachlassen der spezifischen revolutionären Dynamik und der Tatsache, dass sich die nicht geplanten immer wieder auftretenden Ansätze einer gesellschaftlichen Eigenentwicklung als stärker erwiesen als die geplante »Revolution von oben«. Indem Löwenthal den »gesellschaftspolitischen Konflikt zwischen ideologischem Anspruch (>Revolution von oben`) und gesellschaftlicher Wirklichkeit (`Evolution von unten`) entwicklungstheoretisch« analysierte, vermochte er im Rahmen seiner Totalitarismuskonzeption in der Kontinuität des institutionellen Parteiregimes der KPdSU

gleichsam die Diskontinuität ihrer revolutionären Dynamik aufzufangen. Während sich der totalitäre Charakter der Sowjetunion über lange Phasen ihrer Entwicklung dadurch begründet hat(te), daß die KPdSU mit ihrem staatlichen Machtmonopol primär der ideologischen Utopie einer klassenlosen Gesellschaft nachstrebte, gleichwohl aber aus innen- und außenpolitischer Notwendigkeit tatsächlich einer modernen Industriegesellschaft zustrebte, ist im Verlauf der historischen Entwicklung einer erfolgreichen Modernisierung die ideologisch bedingte revolutionäre Dynamik des sowjetischen Totalitarismus erlahmt. ${ }^{60}$

Bei der Analyse dieses Entwicklungsproblems der Sowjetunion, das indes auch für andere kommunistische »Entwicklungsdiktaturen« galt (z. B. China) ${ }^{61}$ ging Löwenthal von drei Hypothesen aus:

Erstens, daß im Verlauf des Entwicklungsprozesses die geplante Revolution von oben in wiederholten Konflikt mit ungeplanter Evolution von unten gerät, die in eine andere Richtung tendiert. Zweitens, daß mit zunehmendem Entwicklungserfolg die Kräfte der Evolution von unten stärker und die Kräfte der Revolution von oben schwächer werden, bis der Prozeß der wiederholten revolutionären Umwälzung seine Schwungkraft einbüßt und schließlich zum Stillstand kommt, weil die Kosten weiterer erzwungener Umwälzung für das Regime selbst - durch Einbuße an wirtschaftlicher Kraft und internationaler Macht - als untragbar empfunden werden. Drittens, daß der Verlust der revolutionären Dynamik, der die totalitären politischen Institutionen ihrer Legiti-

State, in: Ders., The Impact of the Russian Revolution 1917-1967. The Influence of Bolshevism on the World Outside Russia. Introduced by Arnold ]. Toynbee, London/New York/Toronto 1967, S. 274-351; ders., 1917 and After. On the Model of Totalitarian State, in: Encounter 29 (1967), H. 4, S. 21-31; ders., »1917《: an Afterthought on Totalitarian Models, in: Encounter 29 (1967), H. 5, S. 60-64; ders., Von der gelenkten Revolution von oben zur spontanen Evolution von unten, in: Ders./Boris Meissner (Hg.), Sowjetische Innenpolitik. Triebkräfte und Tendenzen, Stuttgart 1968, S. 114-129.

59 Ders., Entwicklung kontra Utopie. Das kommunistische Dilemma, in: Der Monat 22 (1970), H. 266, S. 60-84.

60 Möll, Gesellschaft und totalitäre Ordnung, S. $160 \mathrm{f}$.

61 Vgl. ebd., S. 74-78. 
mation beraubt, zu einem wichtigen Faktor für die Veränderung dieser Institutionen werden muß. ${ }^{62}$

Alle drei Hypothesen hatten sich für Löwenthal im Falle der Sowjetunion bestätigt, weil der Verlauf der historischen Entwicklung des kommunistischen Parteiregimes zeigte, dass sich seine »Funktion und Wertmaßstäbe« gewandelt hatten. Der Verlust der für totalitäre politische Systeme typischen revolutionären Dynamik hatte tatsächlich Veränderungen in der Herrschaftsstruktur zur Folge gehabt; das Parteienmonopol hatte sich wirklich von seinem »totalitären Status « fortentwickelt. ${ }^{63}$ Dieser Prozess hatte für Löwenthal nämlich zur Umkehr der "grundlegende[n] Beziehung zwischen dem monopolistischen Parteiregime und der "gesellschaftlichen Entwicklung« geführt. ${ }^{64}$ Das politische System unterwarf jetzt nicht mehr mit seiner Befehlsgewalt eine »unterentwickelte Gesellschaft zugleich einer forcierten Entwicklung und einer Reihe von Revolutionen von oben«, sondern es musste »auf den Druck reagieren, der von einer zunehmend modernen Gesellschaft « ausging..$^{65}$ Ebendieser Wandel hatte zur Folge, dass "sowohl der Geist als auch die Methoden der Einparteienherrschaft - das Selbstverständnis des Regimes und seine Regierungsweise - tiefgehend verändert worden « sind. ${ }^{66}$

Dieses postrevolutionäre Parteiregime, schrieb er, »kann nicht mehr seine revolutionäre Offensive gegen die Gesellschaft fortsetzen; es will sich nicht auf einen bloßen Ausdruck gesellschaftlicher Kräftekonstellation in einem bestimmten Zeitpunkt reduzieren lassen. So ist es weder totalitär noch demokratisch, sondern autoritär. « ${ }^{67}$

Somit stellte Löwenthal im Rahmen seiner Totalitarismuskonzeption und seines am Sowjetkommunismus gewonnenen dynamischen Totalitarismusbegriffs Anfang 1970 im Monat fest, dass die zeitgenössische nachstalinistische »Monopolpartei-Herrschaft " (Walter Schlangen) angemessen nur als autoritäre und nicht mehr als totalitäre Diktatur klassifiziert werden konnte; insofern kam es in diesem Zusammenhang zur Rückbildung von der Totalitarismus- zur Autoritarismuskonzeption.

Zusammenfassend betrachtet scheinen die löwenthalsche Totalitarismuskonzeption und sein am Herrschaftsphänomen des Sowjetkommunismus gewonnener Totalitarismusbegriff der »totalitären Revolution im Monat nicht nur wissenschaftlicher Motivation geschuldet gewesen zu sein. Den Anspruch, mit seinem dynamischen Totalitarismusbegriff die politischen Systeme des deutschen Nationalsozialismus und des sowjetischen Kommunismus (sowie des chinesischen Kommunismus) gleichzeitig theoretisch zu erfassen, konnte er ausschließlich anhand des »roten « Totalitarismus empirisch belegen; anders gesagt: Die vergleichende Analyse der Verlaufsform der deutschen "Revolution « mit der Russischen Revolution erfolgte ohne eine empirische Prüfung beispielsweise des primär anhand des historischen Tatsachenmaterials des

\footnotetext{
62 Ebd., S. 78.

63 Schlangen, Die Totalitarismus-Theorie, S. 115.

64 Richard Löwenthal, Entwicklung kontra Utopie. Das kommunistische Dilemma, in: Der Monat 22 (1970), H. 266, S. 81.

65 Ebd.

66 Ebd., S. 84.

67 Ebd.
} 
Nationalsozialismus entwickelten Begriffs der "permanenten Revolution«, die Neumann in seiner 1942 erschienenen gleichnamigen Totalitarismusstudie vorlegte.

Löwenthal wandte sich ausdrücklich gegen die Modelle (Arendt, Friedrich/Brzezinski), die den Totalitarismus als ein politisches System definierten, das seinem Wesen nach zu keinen inneren Wandlungen fähig sei. ${ }^{68}$ Sein Aufsatz Totalitäre und demokratische Revolution empfing zweifelsohne seinen >Sinn durch die nicht zuletzt durch die Entwicklungen in Ungarn und Polen 1956 genährte Hoffnung, dass die totalitäre Diktatur in der Sowjetunion erstens nicht die "Sackgasse der Weltgeschichte " sei und zweitens die revolutionäre Dynamik des monopolistischen Parteiregimes zum Stillstand kommen könnte, sodass das Zeitalter des Totalitarismus bzw. der »totalitären Revolution« endlich zu Ende geht. ${ }^{69}$

Gleichwohl hat es den Anschein, als ob es Löwenthal mit seinem am Modell des sowjetischen Kommunismus entwickelten Begriffes der totalitären Herrschaft im Monat nicht zuletzt um den Nachweis seiner zeitgenössischen Bedrohlichkeit für die westlichen Demokratien ging. Denn nachdem die klassischen Totalitarismustheorien den nach Stalins Tod einsetzenden Wandlungsprozess des sowjetischen Herrschaftssystems begrifflich nicht erklären konnten und nicht zuletzt aufgrund der konzeptionellen Schwächen infrage gestellt wurden, gelang es Löwenthal, den Totalitarismusbegriff nur aufrechtzuerhalten, indem er den Anwendungsbereich des Begriffes erweiterte und somit nicht mehr der (Massen-)Terror als unverzichtbares Merkmal einer totalitären Diktatur galt. Vor diesem Hintergrund wird hier die These vertreten, dass die Totalitarismuskonzeption und der -begriff von Löwenthal, nachdem der deutsche Nationalsozialismus nicht mehr existierte, in den Jahren des Kalten Krieges im Monat in der Weise ausgeweitet wurde, dass sie realhistorisch in erster Linie mit der sowjetkommunistischen Herrschaft zur Deckung gebracht werden konnte. Dass er sich erst 1970 im Monat vom Totalitarismusbegriff verabschiedete, um die Sowjetunion im Kontext des entschärften Ost-West-Konfliktes als autoritäres Regime zu charakterisieren, ${ }^{70}$ obwohl bereits 1964 der Nachweis erfolgte, dass ein »bolschewistisches System unter den Bedingungen der Industriegesellschaft [...] eher zu einer autoritären als zu einer totalitären Verfassung « tendierte, ${ }^{71}$ hatte seinen Erklärungsgrund "offenbar allein in der politischen Funktion des Begriffes totalitärer Herrschaft«, wie es Jänicke bereits auch und vor allem mit Blick auf Friedrich feststellte. ${ }^{72}$

68 Ebd., S. 78 (Anm. 9).

$69 \mathrm{Vgl}$. Schulz, Der Begriff des Totalitarismus, S. $449 \mathrm{f}$.

70 Hier sei daran erinnert, dass Ende der 1960er-Jahre vom damaligen deutschen Bundeskanzler mit seiner Ostpolitik eine neue Phase der Entspannungspolitik gegenüber der Sowjetunion und den osteuropäischen Satellitenstaaten eingeleitet wurde, die durch die Unterzeichnung der Moskauer Verträge am 12. August 1970 einen vorläufigen Höhepunkt fand, indem sich die Bundesrepublik und die UdSSR verpflichteten, auf gegenseitige Gewaltanwendung und auf Gebietsansprüche zu verzichten.

71 Peter Christian Ludz, Entwurf einer soziologischen Theorie totalitär verfaßter Gesellschaft, in: Seidel/Jenkner, Wege der Totalitarismus-Forschung, S. 532-559, hier S. 549.

72 Jänicke, Totalitäre Herrschaft, S. 243. Zur politischen Funktion der Totalitarismustheorie in der Zeiten des Kalten Krieges, vgl. grundsätzlich Abbott Gleason, Totalitarianism. The Inner History of the Cold War, New York 1995. 

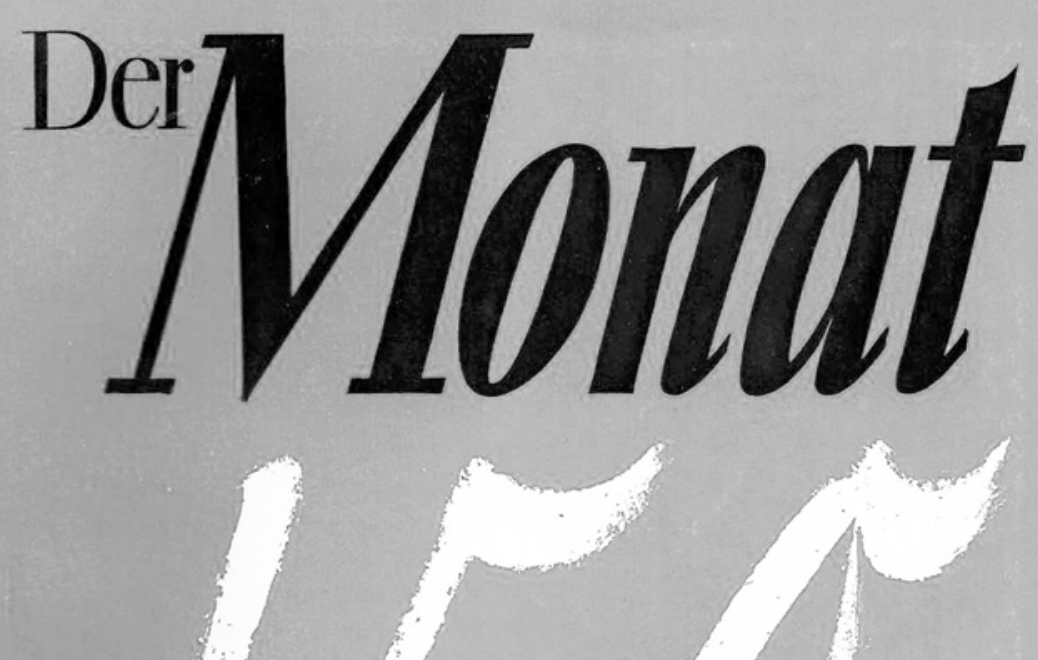

Mit Beiträgen von Karl Jaspers

Raymond Aron / François Bondy

John Strachey / Edouard Roditi

R. Lowenthal und F.R.Allemann

MÄRZ 1961 - HEFT 150

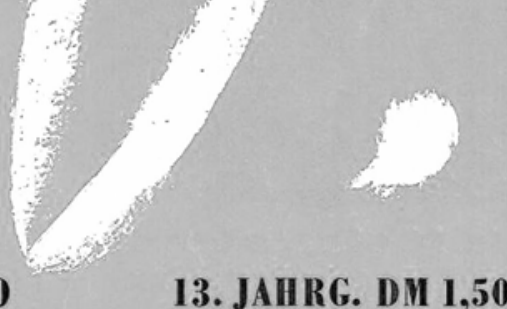

A $4942 \mathrm{E}$

Frontispitz der Ausgabe 150 (1961) 